\author{
Sven Haller \\ Andreas J Bartsch \\ Ernst W Radue \\ Markus Klarhöfer \\ Erich Seifritz \\ Klaus Scheffler
}

\section{Effect of fMRI acoustic noise on non-auditory working memory task: comparison between continuous and pulsed sound emitting EPI}

Received: 17 May 2005

Accepted: 30 September 2005

Published online: 18 November 2005

(C) ESMRMB 2005

S. Haller $(\varangle) \cdot$ E.W. Radue

Institute of Neuroradiology,

Department of Medical Radiology,

University Hospital Basel,

Petersgraben 4,

CH-4031 Basel,

Switzerland

e-mail: shaller@uhbs.ch

Tel.: +41-61-2652525

A.J. Bartsch

Department of Neuroradiology,

University of Würzburg,

Würzburg, Germany

M. Klarhöfer · K. Scheffler

MR Physik, Department of Medical

Radiology,

University Hospital Basel, Basel,

Switzerland

E. Seifritz

University Hospital of Clinical Psychiatry, University of Berne, Berne, Switzerland

\author{
Abstract Conventional blood \\ oxygenation level-dependent \\ (BOLD) based functional magnetic \\ resonance imaging (fMRI) is \\ accompanied by substantial acoustic \\ gradient noise. This noise can \\ influence the performance as well as \\ neuronal activations. Conventional \\ fMRI typically has a pulsed noise \\ component, which is a particularly \\ efficient auditory stimulus. We \\ investigated whether the elimination \\ of this pulsed noise component in a \\ recent modification of \\ continuous-sound fMRI modifies \\ neuronal activations in a cognitively \\ demanding non-auditory working \\ memory task. Sixteen normal \\ subjects performed a letter variant \\ $n$-back task. Brain activity and \\ psychomotor performance was \\ examined during fMRI with \\ continuous-sound fMRI and \\ conventional $\mathrm{fMRI}$. We found \\ greater BOLD responses in bilateral \\ medial frontal gyrus, left middle \\ frontal gyrus, left middle temporal \\ gyrus, left hippocampus, right \\ superior frontal gyrus, right \\ precuneus and right cingulate gyrus
}

with continuous-sound compared to conventional fMRI. Conversely, BOLD responses were greater in bilateral cingulate gyrus, left middle and superior frontal gyrus and right lingual gyrus with conventional compared to continuous-sound fMRI. There were no differences in psychomotor performance between both scanning protocols. Although behavioral performance was not affected, acoustic gradient noise interferes with neuronal activations in non-auditory cognitive tasks and represents a putative systematic confound.

Keywords BOLD - Acoustic noise Working memory · Distraction . Attention
Abbreviations BA: Brodman's area. BOLD: Blood oxygenation level dependent - fMRI: Functional magnetic resonance imaging - GLM: General linear model . TAL: Talairach space - VOI: Volume of interest

\section{Introduction}

Functional magnetic resonance imaging (fMRI) allows the non-invasive assessment of neuronal activation at high spatial and acceptable temporal resolution and has revolutionized neuroimaging. One limitation of fMRI is the substantial acoustic noise emitted during the acquisition of blood oxygenation level-dependent (BOLD) sensitive functional images, which is due to fast magnetic field gradient switches necessary for the generation of images. Depending on the specific sequence parameters, the typical MR gradient noise is characterized by a high- 
frequency sound with a fundamental frequency in the range between 500 and $1000 \mathrm{~Hz}$ and a low-frequency pulse modulation component of about $8-12 \mathrm{~Hz}$. The human auditory cortex is particularly susceptible to stimulus presentation rates in this low range [1-4]. Current solutions to avoid interaction between acoustic scanner noiserelated and experimental stimulus-related brain activity, in particular in the context of auditory fMRI, include sparse sampling $[5,6]$, averaged single trial image sampling [7] or silent gradient switching [8]. These techniques are time-consuming and inefficient in representing time-varying auditory stimuli [9]. Recently, a modified echoplanar imaging (EPI) sequence was introduced, with continuous instead of pulsed auditory noise. This continuous-sound fMRI reduces the baseline BOLD signal in the auditory cortex and enhances the BOLD response $[10,11]$. It was shown that auditory noise not only interacts with auditory stimuli but also increases motor activation and decreases visual activation [12]. Equivalent activation changes in these regions in response to noise were however not present in another study [13]. Behavioral experiments clearly demonstrate that acoustic noise interferes with non-auditory cognitive tasks. The acoustic noise might distract the subject, impeding the direction of selective attention to a given cognitive task. This interference was attributed to attentional effects and distraction, which is present particularly in complex tasks [14]. For instance, playing tape-recorded conventional pulsed fMRI gradient noise while silently recording event-related responses modulated sensory auditory components and additionally, yet to a lesser degree, cognitive processing especially when studying higher cognitive functions [15]. Additionally, a positron emission tomography (PET) study demonstrated that the introduction of previously recorded fMRI noise into the almost silent PET environment modifies neuronal activations in visual mental imagery [16]. There is no direct evidence in the literature that continuous sound is less detrimental for cognition than pulsed sound. Nevertheless, we reason that because pulsed sound is a particularly potent auditory stimulus and activates cortical and subcortical auditory brain structures stronger than continuous sounds $[1,2,17]$, pulsed sound presumably habituates less than continuous sound, and contains more information. Consequently, continuous sound is presumably less distractive.

In the present study we investigated whether cognition and associated neural activity is altered with continuoussound fMRI compared to conventional fMRI with pulsating auditory noise. Since working memory is particularly susceptible to auditory distracting noise [14], we chose a demanding working memory task. We used non-auditory stimuli in order to avoid putative systematic confounds between auditory stimulus presentation and processing and fMRI noise.

\section{Methods}

Subjects

Sixteen subjects (eight females, eight males, mean age and standard deviation, $25.2 \pm 3.9$ years) gave their written informed consent prior to inclusion in the study. Handedness was assessed using an online version of the Edinburgh Handedness Inventory [18], (http://airto.loni.ucla.edu). Subjects were strongly righthanded $(N=14)$, weakly left-handed $(N=1)$ and moderately left-handed $(N=1)$. Subjects had no history of medical, neurological or psychiatric disorders. In order to avoid systematic behavioural confounds between continuous-sound and conventional fMRI, all subjects were familiarized with the task demands using a training program outside the scanner with different stimuli than in the actual experiment. All subjects had to achieve at least $75 \%$ accuracy before participating in the experiment.

\section{Experimental tasks}

Subjects performed a sequential letter task also known as letter variant of an n-back task, which is an established working memory probe [19-23]. In brief, subjects were placed in the scanner and visual stimuli were back-projected onto a semi-translucent canvas during functional imaging. In the control condition 0 back, subjects responded to a single pre-specified target letter (e.g. " $\mathrm{x}$ "). In the active condition 3-back, the target was any letter that was identical to the one presented three items back. Stimuli were pseudorandom sequences of consonants (randomly varying in case), presented in the centre of the visual field (500ms duration, 2500-ms stimulus onset asynchrony). Subjects responded to each stimulus by pressing with the right thumb one button of an MR-compatible hand-held response box for targets (33\% of trials) and another button for non-targets. In each 3back sequence, a number of stimuli were non-target repeats that were included as foils (e.g. 2-back repeats). Conditions were run in blocks of $52.5 \mathrm{~s}$ (21 stimuli). Each condition was repeated five times in pseudo-random order. A delay of $15 \mathrm{~s}$ between blocks was provided as a rest break for subjects and to allow the hemodynamic response to recover from the previous block. Each subject performed two runs. The order of continuous-sound and conventional fMRI runs was fully counterbalanced to avoid order effects.

Image acquisition

Imaging was performed on a $1.5 \mathrm{~T}$ scanner (Sonata, Siemens Medical Solution, Erlangen, Germany). Conventional EPI is composed of slice-selective excitation followed by a read-out train. This read-out train consists of a rapidly switched alternating read gradient and small phase-encoding gradient blips. The resulting gradient noise is thus characterized by a high frequency component of about $500-1000 \mathrm{~Hz}$ from the read-out gradient modulated by a low-frequency component of about $8-12 \mathrm{~Hz}$ generated by the repetition of consecutive slices (assuming a 


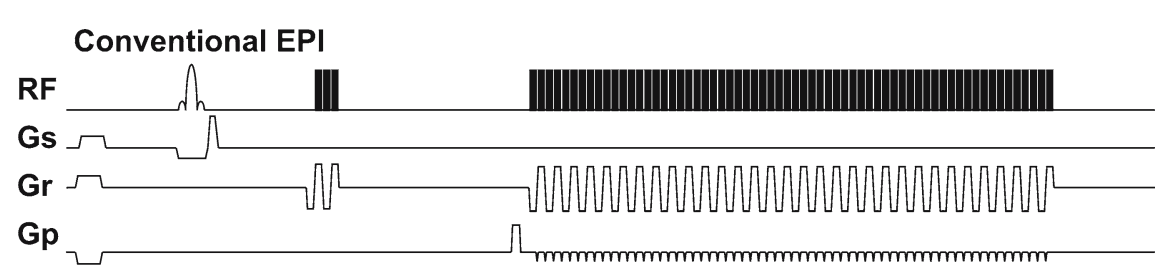

Continuous-sound EPI

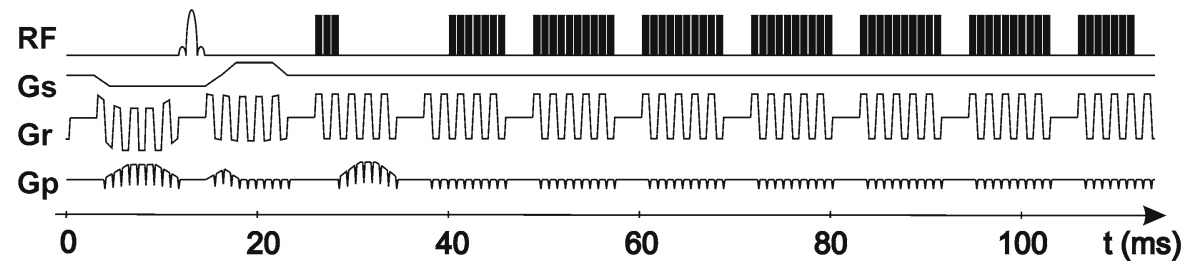

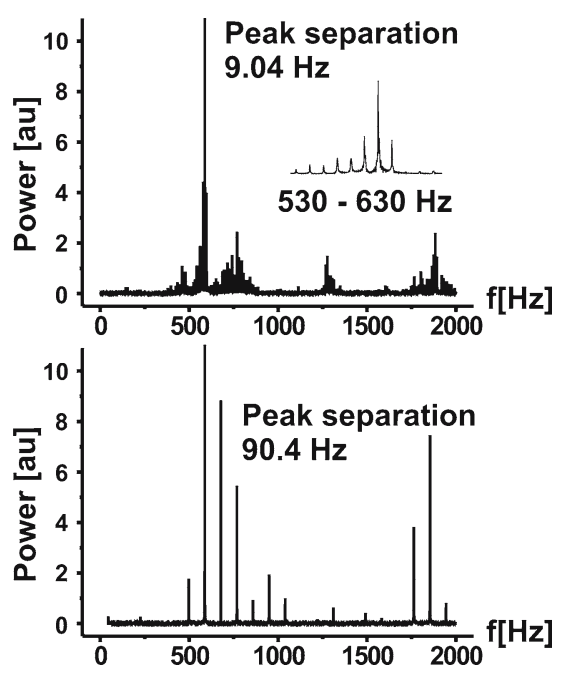

Fig. 1 Sequence diagram and corresponding acoustic noise power spectrum of conventional and continuous-sound echoplanar imaging (EPI). Repitition time (TR) and echotime (TE) are identical for both variants. With a slice acquisition time of $110.6 \mathrm{~ms}$, the acoustic spectrum of conventional EPI shows several side bands separated by $9.4 \mathrm{~Hz}$. Repetition of separated readout blocks for every $11.06 \mathrm{~ms}$ results in a peak separation of $90.4 \mathrm{~Hz}$ for continuous-sound EPI

slice acquisition time of $80-120 \mathrm{~ms})$. In contrast, the continuoussound EPI sequence produces continuous rather than pulsed gradient noise. For continuous-sound EPI, the read-out and phase-encoding train was divided into several separat blocks of five read-out pairs (ten echoes) and ten phase-encoding blips. Consecutive blocks are separated by a period of $2 \mathrm{~ms}$ required for slice-selective excitation at the beginning of the echo train. Additional gradients required for slice selection, phase/read-dephasing, and -spoiling have been implemented with very low gradient amplitudes and long rise and fall times $(2 \mathrm{~ms})$ and were added to the continuously running read-out and phase-encoding blocks $[10,11]$. As a result gradient noise emitted by continuous-sound EPI is composed of a high frequency part consisting of 500$1000 \mathrm{~Hz}$ from the readout gradient modulated by a mediumfrequency component of $70-100 \mathrm{~Hz}$ from repeated blocks of ten echoes, as depicted in Fig. 1. Thus, continuous-sound fMRI gradient noise is perceived as continuous background sound. However, the overall sound pressure level of both fMRI variants is similar.

Other parameters such as bandwidth, echo time (TE), repetition time (TR) and spatial resolution were set identical for continuous-sound and conventional fMRI. The matrix size was $64 \times 64\left(\right.$ FOV $\left.192 \times 192 \mathrm{~mm}^{2}\right)$ and 25 slices were acquired $(4.5 \mathrm{~mm}$ slice thickness, $1 \mathrm{~mm}$ gap) that covered the whole brain. The resulting resolution was $3 \times 3 \times 5.5 \mathrm{~mm}^{3}$. TR was $2.32 \mathrm{~s}(110.6 \mathrm{~ms}$ per slice), flip angle $90^{\circ}$, and TE $61 \mathrm{~ms}$. The first three volumes were discarded from further analysis to avoid non-steadystate saturation effects. After functional scanning, high-resolution data was acquired ( $1 \mathrm{~mm}$ Iso-Voxel T1MPRage, Matrix $256 \times 256,176$ slices) and used for co-registration and normalization.
Image analysis

Anatomical and functional images were analyzed using BrainVoyager QX (Brain Innovation, Maastricht, The Netherlands). Anatomical scans were segmented for identification of the whitegrey matter boundary used for cortical surface reconstruction and cortex-based statistics. Pre-processing of functional time series consisted of three-dimensional motion correction, interscan slice time correction, gaussian spatial filtering (full width half maximum $4 \mathrm{~mm}$ ), high-pass temporal filtering (three cycles per time course) and transformation into standard space [24]. The main effect for the comparison of 3-back versus 0 -back was examined using two separate general linear model (GLM) analyses for continuous-sound and conventional fMRI. The difference between continuous-sound (3-back minus 0-back) versus conventional fMRI (3-back minus 0-back) was determined in an additional GLM. Because we expected a small difference between continuous-sound and conventional fMRI, we performed fixed effects GLM analyses. The results therefore refer to the investigated sample. The differential effect between continuoussound and conventional fMRI was modeled on the second level, and statistical thresholding corrected for multiple comparisons based on the false discovery rate (FDR) [25] at a false-positive probability of $q(\mathrm{FDR})<0.01$. This corresponded to $p$ (uncorrected $)<0.0002$ and $t>3.70$. Spatial extent was $250 \mathrm{~mm}^{3}$. The same thresholds $\left(t>3.70,250 \mathrm{~mm}^{3}\right)$ were applied to the individual GLMs examining main effects of activity measured using continuous-sound and conventional fMRI and corresponded to $q(\mathrm{FDR})<0.001$.

\section{Behavioral data}

Response latency and response accuracy, i.e., the proportion of correct responses, were determined using E-Studio (www.pstnet.com). The average response latency and accuracy were compared between continuous-sound and conventional fMRI and 
between first and second run using repeated measure ANOVA tests and pair wise Bonferroni's multiple comparison tests in GraphPad PRISM (www.graphpad.com).

\section{Results}

\section{Behavioral data}

The average response times for continuous-sound fMRI were $577 \pm 79 \mathrm{~ms}$ (mean $\pm \mathrm{SD}$ ) for 0-back and $863 \pm 129 \mathrm{~ms}$ for 3-back task. Correspondingly, response times for conventional fMRI were $575 \pm 68 \mathrm{~ms}$ and $894 \pm 126 \mathrm{~ms}$ for 0 -back and 3-back, respectively. The comparison of response times between 0-back and 3-back and between continuous-sound and conventional fMRI revealed a significant group difference (repeated measures ANOVA $p<0.0001$ ). There was no significant difference between continuous-sound and conventional fMRI. The response latency increased from 0-back to 3-back (Bonferroni's multiple comparison tests $p<0.001$ for continuoussound and conventional fMRI). The average accuracy for continuous-sound fMRI was $98.5 \pm 2.1 \%$ and $82.9 \pm 7.9 \%$ for zero-back and three-back, respectively. The average accuracy for conventional fMRI was $98.8 \pm 1.4 \%$ and $84.3 \pm 6.9 \%$ for 0 -back and 3-back, respectively. The comparison of response accuracy between 0-back and 3-back and between both fMRI variants revealed a significant group difference (repeated measures ANOVA $p<0.0001$ ). No significant difference was present between both fMRI variants. The response accuracy decreased from 0 -back to 3-back (Bonferroni's multiple comparison tests $p<0.001$ for continuous-sound and conventional fMRI).

Additionally, the first and second runs were compared. There were no significant differences in accuracy (first run $98.6 \pm 1.8 \%$ 0-back, $82.4 \pm 6.4 \%$ 3-back; second run $98.7 \pm 1.7 \% 0$-back, $84.8 \pm 8.2 \% 3$-back) or in response times (first run $585.8 \pm 76.5 \mathrm{~ms} \mathrm{0}$-back, $898.9 \pm 112.6 \mathrm{~ms} \mathrm{3-}$ back; second run $566.7 \pm 69.6 \mathrm{~ms} 0$-back, $858.8 \pm 139.6 \mathrm{~ms}$ 3 -back) between first and second run. There were however significant decrease in accuracy and significant increase in response times from 0-back to 3-back (repeated measures ANOVA $p<0.0001$, Bonferroni's multiple comparison tests $p<0.001$ for all latter pair wise comparisons).

\section{Neuronal activation}

First, the main effect for the comparison 3-back versus 0 -back was calculated separately for continuous-sound and conventional fMRI. Both fMRI variants were able to detect similar and remarkably symmetrical activation clusters in the memory task including bilateral superior, medial, middle and inferior frontal gyrus, anterior and posterior cingulate gyrus, insula, cuneus, precuneus, superior and inferior parietal lobule, superior occipital gyrus, middle and superior temporal gyrus, fusiform gyrus, lingual gyrus and cerebellum. These activation clusters are illustrated in Fig, 2 and listed in detail in Table 1.

Second, the difference between continuous-sound fMRI (3-back minus 0-back) and conventional fMRI (3back minus 0-back) was analyzed to assess the effect of acoustic fMRI noise. Stronger activation for continuous-sound compared to conventional fMRI was found in bilateral medial frontal gyrus, left anterior inferior middle frontal gyrus, left middle temporal gyrus, left hippocampus, right superior frontal gyrus, right precuneus and right cingulate gyrus. Conversely, stronger activation for conventional versus continuous-sound fMRI was found in bilateral cingulate gyrus, left posterior superior middle and superior frontal gyrus and right lingual gyrus. All activation clusters are illustrated in Fig. 3 and listed in Table 2.

\section{Discussion}

The present study investigates the effect of acoustic fMRI noise on BOLD activations evoked during a demanding non-auditory working memory task. Acoustic scanner noise might distract the subject, impeding the direction of selective attention to a given cognitive task. The majority of fMRI experiments are based on conventional EPI sequences. This conventional fMRI typically has a pulsed noise component of about $8-12 \mathrm{~Hz}$, which is a particularly efficient auditory stimulus [2,17]. Stimulus repetition rates in that range are also potent stimuli in other sensory systems [26], suggesting that pulsed noise might affect cognition more than continuous noise. We used a novel continuous-sound fMRI, which implements a quasi-continuous gradient switch pattern. This evokes a quasi continuous scanner acoustic noise and lower baseline activity produced by the scanner noise $[10,11]$. A putative distracting effect of MR gradient noise is presumably more pronounced in a cognitively demanding experiment. Because working memory is particularly susceptible to auditory distracting noise [14], we chose an established and challenging working memory letter $n$ back task, also known as sequential letter task [19-23]. In the active 3-back condition a target is any letter that is equivalent to the letter three items back. The sequence of letters must be maintained and updated continuously in working memory, which poses a high load on working memory. In the control 0-back condition, the demand on working memory is neglible because the target simply is a predefined letter. In order to avoid systematic confounds related to interference of acoustic scanner noise and stimulus presentation [9], we chose a non-auditory experiment. Since the MR gradient noise is only one disturbing factor among others, like being in an unknown environment inside a MR scanner, we assume that the 


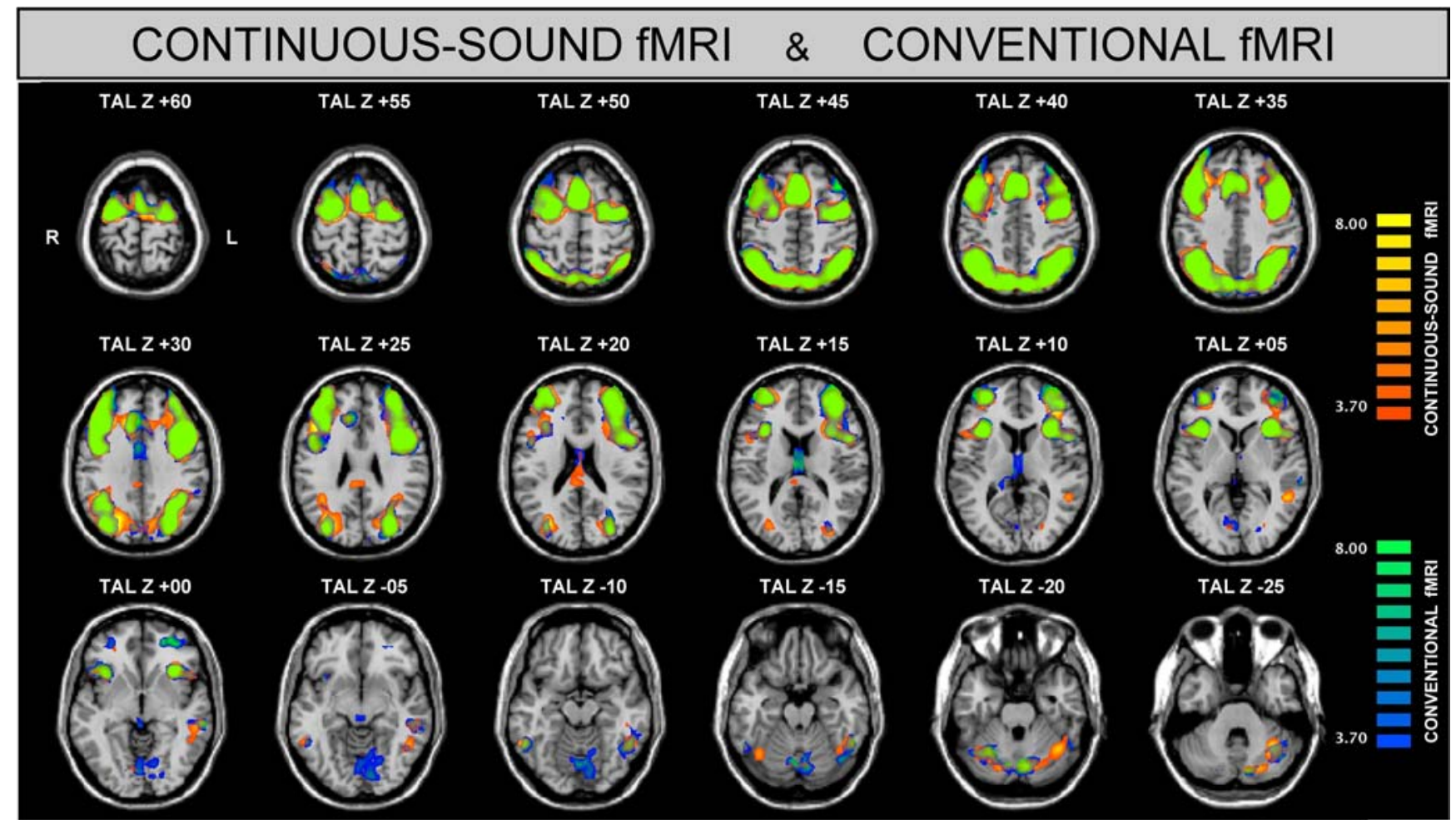

Fig. 2 The main effect 3-back versus 0-back is illustrated separately for continuous-sound fMRI (orange to yellow) and conventional fMRI (blue to green) based on fixed effect general linear model analyses with statistical threshold of $q<0.001$ corrected (false discovery rate, $F D R$ ) and extent threshold of $250 \mathrm{~mm}^{3}$. TAL Talairach coordinates, axial slices in radiologic convention with right hemisphere displayed on left hand side

differences between continuous-sound and conventional fMRI are considerably smaller than differences in primary auditory processing $[10,11]$. In particular, we tested the hypothesis that continuous-sound fMRI modifies neuronal activations compared to conventional fMRI.

The analysis of the main effect (3-back vs. 0-back) revealed similar BOLD activation patterns for both fMRI variants. The resulting activation clusters include bilateral superior, medial, middle and inferior frontal gyrus, bilateral cingulate gyrus, insula, cuneus, precuneus, superior and inferior parietal lobule, bilateral superior and middle temporal gyrus, bilateral superior occipital gyrus, fusiform gyrus, lingual gyrus and cerebellum. These results are consistent with previous neuroimaging studies implementing letter $n$-back tasks [19-23].

The effect of auditory fMRI noise on cognitive processing was assessed by the comparison of the main effect (3-back vs. 0-back) between continuous-sound and conventional fMRI. This comparison yielded several significant differences in neuronal activations, including frontal, temporal, cingulate and lingual gyrus, hippocampus and precuneus. The primary intention of the present investigation was to test whether the modification of background

noise characteristics between continuous-sound and conventional fMRI alters neuronal activations, which we could unambiguously document. In the next step, it is possible to infer putative cognitive mechanisms underlying the differences between both fMRI variants based on the interpretation of the observed activation patterns.

Continuous-sound compared to conventional fMRI evoked significant differences in several regions pertinent for working memory formation including bilateral medial frontal gyrus, left middle frontal gyrus, left middle temporal gyrus, left hippocampus, right superior frontal gyrus, right precuneus and right cingulate gyrus. We consider the acoustic MR gradient noise as distraction stimulus, which poses effort on selective attention to focus on the task of interest. We therefore relate the current activations to other neuroimaging experiments investigating the effect of distraction and divided attention. One group of relevant studies for comparison is dichotic listening tasks. In these experiments, different auditory stimuli are presented simultaneously in both ears. Prior knowledge that the stimulus of interest is presented to one ear allows directing attention to that ear. It was shown that focused attention compared to divided attention is associated with stronger activations in right precuneus [27], consistent with the presented results. Very similar activation in the right precuneus was also observed in a more recent dichotic listening task [28]. Remarkably, this study additionally revealed activations in left hippocampal region and left inferior frontal activations in those trials where the target was correctly detected. We reason that 
Table 1 Supra-threshold activation clusters for the comparison 3-back minus 0-back sequential letter task for continuous-sound functional magnetic resonance imaging (fMRI) and conventional fMRI

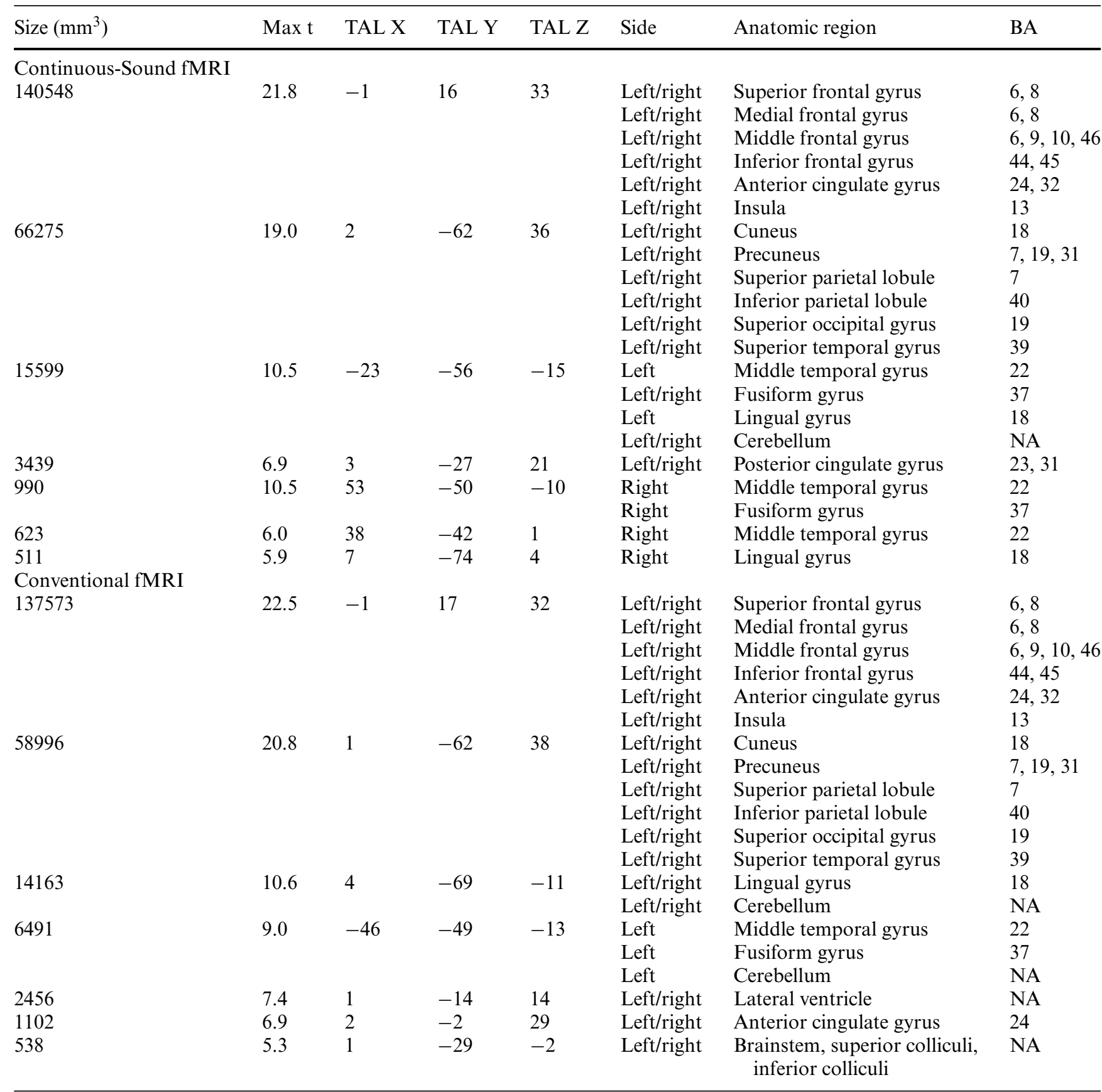

Activations were calculated based on fixed effect general linear models. The statistical threshold was $q($ FDR $)<0.001$ corrected (false discovery rate)

The spatial threshold was $250 \mathrm{~mm}^{3}$. Size of activation cluster in $\mathrm{mm}^{3}$, maximum $t$-value within the activation cluster, center of gravity in Talairach (TAL) coordinates, side, anatomic region and Brodman's area (BA) are listed 
Table 2 Supra-threshold activation clusters for the differential analysis continuous-sound fMRI (3-back-0-back) versus conventional fMRI (3-back-0-back)

\begin{tabular}{|c|c|c|c|c|c|c|c|}
\hline Size $(\mathrm{mm} 3)$ & Max $t$ & TAL X & TAL Y & TAL Z & Side & Anatomic region & BA \\
\hline \multicolumn{8}{|c|}{ Continuous-Sound fMRI > Conventional fMRI } \\
\hline 634 & 5.8 & 19 & -65 & 28 & Right & Precuneus & 18 \\
\hline 443 & 5.4 & 17 & 45 & 18 & Right & $\begin{array}{l}\text { Medial frontal gyrus } \\
\text { Superior frontal gyrus }\end{array}$ & $\begin{array}{l}9 \\
10\end{array}$ \\
\hline 406 & 6.4 & 22 & 31 & 0 & Right & Cingulate gyrus & 24 \\
\hline 324 & 5.4 & -33 & 39 & 11 & Left & Anterior inferior middle frontal gyrus & 10 \\
\hline 289 & 4.7 & -3 & -12 & 59 & Left & Medial frontal gyrus & 6 \\
\hline 275 & 6.4 & -23 & -8 & -22 & Left & Hippocampus & NA \\
\hline 262 & 4.8 & -48 & -47 & 1 & Left & Middle temporal gyrus & 22 \\
\hline \multicolumn{8}{|c|}{ Conventional fMRI > Continuous-Sound fMRI } \\
\hline 1073 & -7.5 & -32 & 44 & -1 & Left & Middle frontal gyrus & 10 \\
\hline 399 & -5.2 & -1 & -5 & 31 & Left & Cingulate gyrus & 24 \\
\hline 362 & -5.1 & -30 & 55 & 14 & Left & Superior frontal gyrus & 10 \\
\hline 357 & -5.9 & 6 & 20 & 19 & Right & Anterior cingulate & $24 / 33$ \\
\hline 351 & -6.3 & -45 & 12 & 39 & Left & Posterior superior middle frontal gyrus & 8 \\
\hline 327 & -6.4 & 1 & -15 & 14 & Right & Lateral ventricles & NA \\
\hline 262 & -5.2 & 2 & -70 & -6 & Right & Lingual gyrus & 18 \\
\hline
\end{tabular}

Activations were calculated based on fixed effect general linear models

The statistical threshold was $q(\mathrm{FDR})<0.01$ corrected (false discovery rate)

The spatial threshold was $250 \mathrm{~mm}^{3}$

Size of activation cluster in $\mathrm{mm}^{3}$, maximum $t$-value within the activation cluster, center of gravity in Talairach (TAL) coordinates, side, anatomic region and Brodman's area (BA) were listed

the target is correctly identified in those trials where the attention was successfully directed to the task of interest, i.e. the distraction had little effect. Consequently, this observation corresponds to the hypothesized reduced distraction of continuous-sound fMRI compared to conventional $\mathrm{fMRI}$ in the present investigation.

On the other hand, BOLD response was greater with conventional compared to continuous-sound fMRI in bilateral cingulate gyrus, left middle and superior frontal gyrus and right lingual gyrus. In line with the presented results, activation in the cingulate cortex was present in a virtually silent positron emission tomography study (PET) of visual mental imagery in response to the introduction of fMRI-like auditory noise [16]. A key role in evaluative processes required to solve complex tasks was attributed to this area $[29,30]$, which is presumably more important in the presence of disrupting auditory noise [16]. Further, cingulate cortex activation was present as a consequence of distraction implemented as verbal attention task during a painful cold pressor test [31].

According to our expectations, no auditory activations were present in the difference analysis between both fMRI variants. Within each fMRI variant, the auditory noise is the same for 0-back and 3-back. Therefore, there is no consequent auditory activation in the difference analysis between 3-back and 0-back, which is the basis for the comparison between the fMRI variants. The absence of auditory activations supports our assumption that the observed differences in neuronal activations between both fMRI variants are non-auditory cognitive consequences in response to the modified noise characteristic and not primary auditory differences.

In order to avoid differences in behavioral performance between continuous-sound and conventional fMRI, subjects had to perform extensive training prior to functional scanning. Such systematic behavioral differences might act as systematic confound impeding the interpretation of putative BOLD differences between continuoussound and conventional fMRI. The absence of behavioral differences between continuous-sound and conventional fMRI as well as between first and second run despite the observed BOLD differences excludes putative behaviorrelated systematic confounds between both fMRI variants and supports the validity of the presented results.

We reason that the most likely origin of the observed neuronal differences between continuous-sound and conventional fMRI is related to distraction. This assumption needs validation in future studies.

The present investigation might be related to a recent fMRI study with similar study design, which investigated the effect of increasing fMRI acoustic noise in a working memory $n$-back task [32]. A conventional "quiet" EPI 


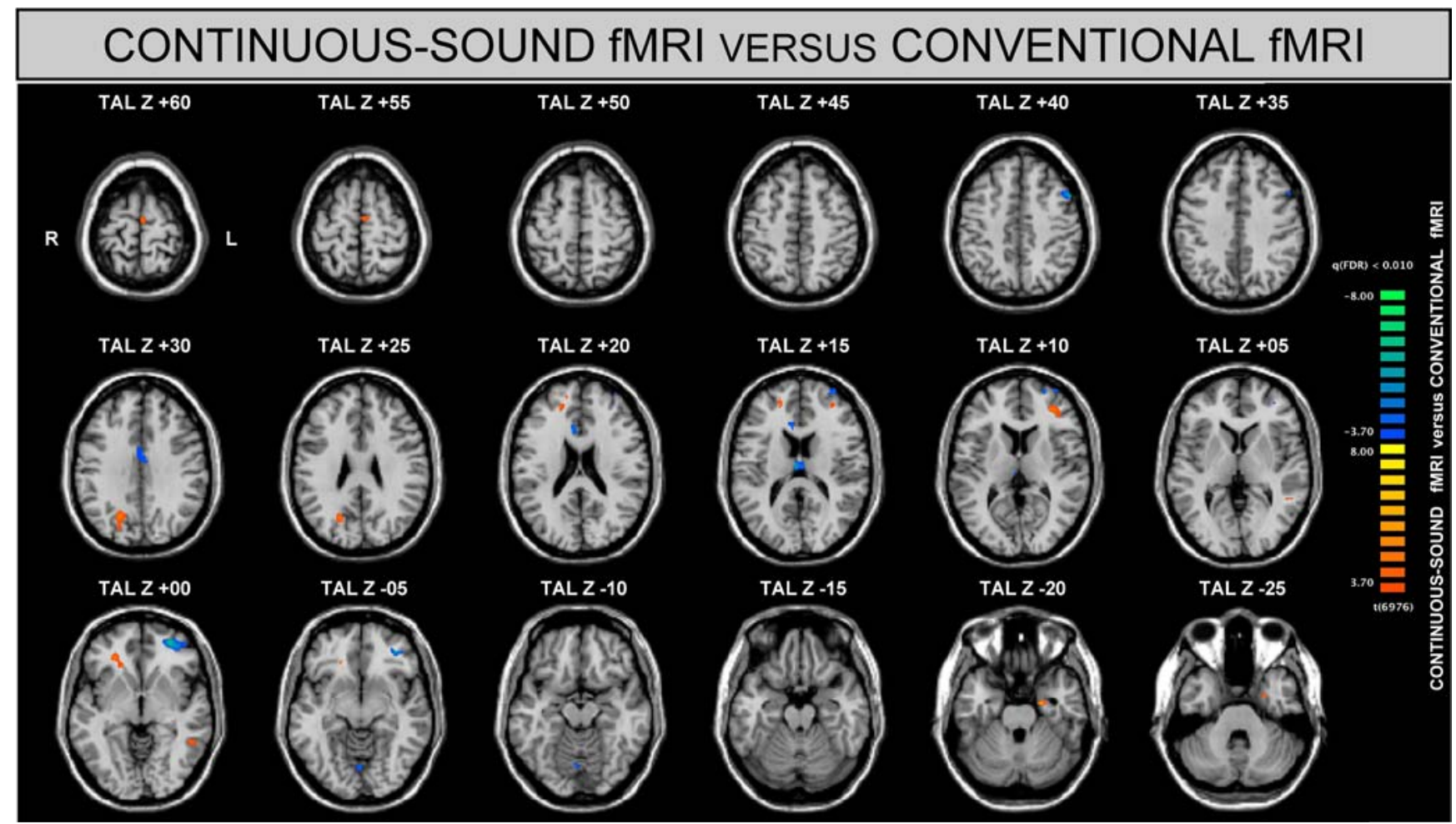

Fig. 3 The difference between continuous-sound fMRI and conventional fMRI was estimated based on a fixed effect general linear model analysis with statistical threshold of $q<0.01$ corrected (false discovery rate, $F D R$ ) and extent threshold of $250 \mathrm{~mm}^{3}$. Stronger activations for continuous-sound fMRI compared to conventional fMRI are orange to yellow, stronger activations for conventional fMRI versus continuous-sound fMRI are blue to green. TAL Talairach coordinates, axial slices in radiologic convention with right hemisphere displayed on left hand side

pulse sequence was compared to a "loud" EPI sequence with a four-fold (12 dBA) increase in sound pressure level (SPL) utilizing resonant vibration modes of the gradient coil. In the present investigation, there is no difference in SPL between continuous-sound and conventional fMRI. All subjects perceived the pulsating noise of the conventional fMRI as less pleasant and more distractive compared to the continuous-sound fMRI. There were no differences in reaction time or accuracy between "loud" and "quiet" in the study by Tomasi et al. [32] or between continuous-sound and conventional fMRI in the present investigation. If we consider "loud" more distractive than "quiet" and conventional fMRI more distractive than continuous-sound fMRI, we may compare both studies. The difference between "loud" and "quiet" remarkably resembles the difference between conventional and continuous-sound fMRI, and includes in both investigations increased activations in superior and middle frontal gyri, lingual gyrus and decreased activation in cingulate gyrus.
The present results thus show that not only sound pressure [32] but also fMRI noise characteristics are putative systematic confounds between different pulse sequences, field strengths and manufacturers even for non-auditory fMRI experiments.

One issue complicating the interpretation of the present investigation is that the interaction of distraction and neuronal activations remains controversial. On the one hand, it is possible that distraction reduces neuronal activations because attention is reduced and directed towards another task [33]. On the other hand it is also possible that distraction increases neuronal activation because it takes more cognitive effort to perform the task of interest [16]. A mixture of both effects depending on the brain region is also possible, as suggested by the current investigation. Because of this remaining uncertainty regarding the interaction of acoustic noise and neuronal activations, we reason that reduction of MR gradient noise reduces auditory distraction and consequently avoids putative systematic confounds.

We conclude that the auditory fMRI noise characteristics interfere with neuronal activations even during nonauditory cognitive tasks and thus are putative systematic confounds.

Acknowledgements This work was supported in part by the Swiss National Science Foundation grants\#PP00B-103012 and\#PP00B68783 


\section{References}

1. Harms MP, Melcher JR (2002) Sound repetition rate in the human auditory pathway: representations in the waveshape and amplitude of fMRI activation. J Neurophysiol 88(3):1433-1450

2. Giraud AL, Lorenzi C, Ashburner J, Wable J, Johnsrude I, Frackowiak R, Kleinschmidt A (2000) Representation of the temporal envelope of sounds in the human brain. J Neurophysiol 84(3):1588-1598

3. Bandettini PA, Jesmanowicz A, Van Kylen J, Birn RM, Hyde JS (1998) Functional MRI of brain activation induced by scanner acoustic noise. Magn Reson Med 39(3):410-416

4. Seifritz E, Di Salle F, Esposito F, Bilecen D, Neuhoff JG, Scheffler K (2003) Sustained blood oxygenation and volume response to repetition rate-modulated sound in human auditory cortex. Neuroimage 20(2):1365-1370

5. Scheffler K, Bilecen D, Schmid N, Tschopp K, Seelig J (1998) Auditory cortical responses in hearing subjects and unilateral deaf patients as detected by functional magnetic resonance imaging. Cereb Cortex 8(2):156-163

6. Hall DA, Haggard MP, Akeroyd MA, Palmer AR, Summerfield AQ, Elliott MR, Gurney EM, Bowtell RW (1999) "Sparse" temporal sampling in auditory fMRI. Hum Brain Mapp 7(3):213-223

7. Belin P, Zatorre RJ, Hoge R, Evans AC, Pike B (1999) Event-related fMRI of the auditory cortex. Neuroimage 10(4):417-429

8. Loenneker T, Hennel F, Ludwig U, Hennig J (2001) Silent BOLD imaging. Magma 13(2):76-81

9. Amaro E, Jr, Williams SC, Shergill SS, $\mathrm{Fu} \mathrm{CH}$, MacSweeney M, Picchioni MM, Brammer MJ, McGuire PK (2002) Acoustic noise and functional magnetic resonance imaging: current strategies and future prospects. J Magn Reson Imaging 16(5):497-510

10. Seifritz E, Di Salle F, Esposito F, Herdener M, Neuhoff JG, Scheffler K (2005) Enhancing BOLD response in auditory system by neurophysiologically tuned fMRI sequence. Neuroimage (accepted)

11. Scheffler K, Seifritz E (2004) Doubling BOLD signal in auditory cortex by acoustic modification of echoplanar sequence. In: ISMRM, Kyoto
12. Cho ZH, Chung SC, Lim DW, Wong EK (1998) Effects of the acoustic noise of the gradient systems on fMRI: a study on auditory, motor, and visual cortices. Magn Reson Med 39(2):331-335

13. Elliott MR, Bowtell RW, Morris PG (1999) The effect of scanner sound in visual, motor, and auditory functional MRI. Magn Reson Med 41(6):1230-1235

14. Banbury SP, Macken WJ, Tremblay S, Jones DM (2001) Auditory distraction and short-term memory: phenomena and practical implications. Hum Factors 43(1):12-29

15. Novitski N, Anourova I, Martinkauppi S, Aronen HJ, Naatanen R, Carlson S (2003) Effects of noise from functional magnetic resonance imaging on auditory event-related potentials in working memory task. Neuroimage 20(2):1320-1328

16. Mazard A, Mazoyer B, Etard O, Tzourio-Mazoyer N, Kosslyn SM, Mellet E (2002) Impact of fMRI acoustic noise on the functional anatomy of visual mental imagery. J Cogn Neurosci 14(2):172-186

17. Seifritz E, Esposito F, Hennel F, Mustovic H, Neuhoff JG, Bilecen D, Tedeschi G, Scheffler K, Di Salle F (2002) Spatiotemporal pattern of neural processing in the human auditory cortex. Science 297(5587):1706-1708

18. Oldfield RC (1971) The assessment and analysis of handedness: the Edinburgh inventory. Neuropsychologia 9(1):97-113

19. Braver TS, Cohen JD, Nystrom LE, Jonides J, Smith EE, Noll DC (1997) A parametric study of prefrontal cortex involvement in human working memory. Neuroimage 5(1):49-62

20. Jansma JM, Ramsey NF, Coppola R, Kahn RS (2000) Specific versus nonspecific brain activity in a parametric N-back task. Neuroimage 12(6):688-697

21. Callicott JH, Mattay VS, Bertolino A, Finn K, Coppola R, Frank JA, Goldberg TE, Weinberger DR (1999) Physiological characteristics of capacity constraints in working memory as revealed by functional MRI. Cereb Cortex 9(1):20-6

22. D'Esposito M, Aguirre GK, Zarahn E, Ballard D, Shin RK, Lease J (1998) Functional MRI studies of spatial and nonspatial working memory. Brain Res Cogn Brain Res 7(1):1-13

23. Smith EE, Jonides J, Marshuetz C, Koeppe RA (1998) Components of verbal working memory: evidence from neuroimaging. Proc Natl Acad Sci USA 95(3):876-882

24. Talairach J, Tournoux P (1988) Co-planar stereotaxic atlas of the human brain. Thieme, New York

25. Genovese CR, Lazar NA, Nichols T (2002) Thresholding of statistical maps in functional neuroimaging using the false discovery rate. Neuroimage 15(4):870-878

26. Fox PT, Raichle ME (1984) Stimulus rate dependence of regional cerebral blood flow in human striate cortex demonstrated by positron emission tomography. J Neurophysiol 51(5):1109-1120

27. Hugdahl K, Law I, Kyllingsbaek S, Bronnick K, Gade A, Paulson OB (2000) Effects of attention on dichotic listening: an 15O-PET study. Hum Brain Mapp 10(2):87-97

28. Pollmann S, Lepsien J, Hugdahl K, von Cramon DY (2004) Auditory target detection in dichotic listening involves the orbitofrontal and hippocampal paralimbic belts. Cereb Cortex 14(8):903-913

29. MacDonald AW, Cohen JD, Stenger VA, Carter CS (2000) Dissociating the role of the dorsolateral prefrontal and anterior cingulate cortex in cognitive control. Science 288(5472):1835-1838

30. Carter CS, Braver TS, Barch DM, Botvinick MM, Noll D, Cohen JD (1998) Anterior cingulate cortex, error detection, and the online monitoring of performance. Science 280(5364): 747-749

31. Frankenstein UN, Richter W, McIntyre MC, Remy F (2001) Distraction modulates anterior cingulate gyrus activations during the cold pressor test. Neuroimage 14(4): 827-836

32. Tomasi D, Caparelli EC, Chang L, Ernst T (2005) fMRI-acoustic noise alters brain activation during working memory tasks. Neuroimage 27:377

33. Arthurs OJ, Johansen-Berg H, Matthews PM, Boniface SJ (2004) Attention differentially modulates the coupling of fMRI BOLD and evoked potential signal amplitudes in the human somatosensory cortex. Exp Brain Res 157(3):269-274 